\title{
Development and preliminary evaluation of a novel low cost VR- based upper limb stroke rehabilitation platform using Wii technology
}

Emmanuel Tsekleves ${ }^{1}$, Ioannis Paraskevopoulos ${ }^{2}$, Alyson Warland ${ }^{3}$, Cherry Kilbride ${ }^{3}$

${ }^{1}$ ImaginationLancaster, Design, LICA, Lancaster University, LICA Building, Bailrigg,

Lancaster University, Lancaster, LA14YW, UK, e.tsekleves@lancaster.ac.uk, +44 (0)1524-

510794 (corresponding author)

${ }^{2}$ School of Engineering and Design, Brunel University, London UB8 3PH, UK

${ }^{3}$ School of Health Sciences \& Social Care, Brunel University, London UB8 3PH, UK

Products and Devices

\begin{abstract}
Purpose: This paper proposes a novel system (using the Nintendo Wii remote) that offers customised, non-immersive, virtual reality-based, upper-limb stroke rehabilitation and reports on promising preliminary findings with stroke survivors.

Method: The system novelty lies in the high accuracy of the full kinematic tracking of the upper limb movement in real-time, offering strong personal connection between the stroke survivor and a virtual character when executing therapist prescribed adjustable exercises/games. It allows the therapist to monitor patient performance and to individually calibrate the system in terms of range of movement, speed and duration.

Results: The system was tested for acceptability with three stroke survivors with differing levels of disability. Participants reported an overwhelming connection with the system and avatar. A two-week,
\end{abstract}


single case study with a long-term stroke survivor showed positive changes in all four outcome measures employed, with the participant reporting better wrist control and greater functional use. Activities, which were deemed too challenging or too easy were associated with lower scores of enjoyment/motivation, highlighting the need for activities to be individually calibrated.

Conclusions: Given the preliminary findings, it would be beneficial to extend the case study in terms of duration and participants and to conduct an acceptability and feasibility study with community dwelling survivors.

Keywords: Virtual Reality, Stroke Rehabilitation, Motion Capture, Nintendo Wii, Games, Feedback

\section{Implications for Rehabilitation}

- Low-cost, off-the-shelf game sensors, such as the Nintendo Wii remote, are acceptable by stroke survivors as an add-on to upper limb stroke rehabilitation but have to be bespoked to provide high-fidelity and real-time kinematic tracking of the arm movement.

- Providing therapists with real-time and remote monitoring of the quality of the movement and not just the amount of practice, is imperative and most critical for getting a better understanding of each patient and administering the right amount and type of exercise.

- The ability to translate therapeutic arm movement into individually calibrated exercises and games, allows accommodation of the wide range of movement difficulties seen after stroke and the ability to adjust these activities (in terms of speed, range of movement and duration) will aid motivation and adherence - key issues in rehabilitation.

- With increasing pressures on resources and the move to more community-based rehabilitation, the proposed system has the potential for promoting the intensity of practice necessary for recovery in both community and acute settings. 


\section{Introduction}

Stroke is a leading cause of long term disability worldwide including in the United Kingdom (UK) where an estimated 110,000 new strokes occur each year, leading to an annual economic burden of more than $£ 2.8$ billion [1]. Following stroke, $70 \%$ of people experience arm weakness, with only 5$20 \%$ regaining full function [2]. Treatment interventions shown to be most effective are characterised by high intensity, repetitive and task specific properties, but there are resource challenges in providing this level of intervention. Stroke rehabilitation can be a long process; as such, much of it takes place in the home setting. However adherence to home exercise programmes (HEPs) is poor with a recent study finding $79 \%$ of patients in the community stopped doing their HEP as prescribed after two days [3]. A perceived lack of support from physiotherapists was the most frequently mentioned factor associated with lack of compliance. Boredom, de-motivation and depression also play a part in patients not engaging with exercise [3].

Several VR-based stroke rehabilitation interventions using robotic systems and custom made inertial measurement units (IMUs) have been proposed over the years [4]-[7]. However, many of the current interventions in rehabilitation are either very expensive (i.e. robot-aided systems) or not suitable for use at home. The innovative use of the Nintendo Wii could present a way to enhance community based stroke rehabilitation of the arm by providing a motivating and cost-effective way of exercising that could be remotely monitored and exercises adjusted as required by a physiotherapist [8]. To this end, a 15-month exploratory research study, known as Research in Wii Rehabilitation (ReWiiRe ${ }^{1}$ ), was undertaken in London, UK, in five National Health Service (NHS) sites to investigate the patient and therapist experience of using the Wii as an adjunct to physiotherapy [9]. This paper details how data from this study were used to inform the design and development of a customised VR-based prototype platform for rehabilitation of the arm after stroke. The platform employs the Nintendo Wii remote and an open source 3D authoring software with a game engine. Firstly, a comprehensive review of the literature is presented to foreground the development work that is the focus of this paper. This is followed by an overview of the architecture of the proposed developed system. Then, results from a formal evaluation of the system are provided and discussed before concluding comments are made.

\footnotetext{
${ }^{1}$ For more information, see http://www.rewiire.org.uk
} 


\section{Literature Review}

A number of recent literary reviews have investigated the experimental use of the Nintendo Wii in different clinical settings and collected patient and therapist responses on its use [10]-[13]. These highlight the importance of promoting better accessibility and more widespread use of affordable home-based systems, which on one hand motivate clinical users and on the other, provide the required motion-sensing fidelity needed to offer VR based rehabilitation. A number of issues with the commercial Nintendo Wii system as well as currently available Wii-based VR interventions have been reported:

- Accurate tracking of the patient through the provision of full kinematic tracking of arm movement in real-time is one of the primary challenges [14].

- The facility for the therapist to remotely monitor and interact with the patient (and vice versa) in line with activity and progress [11].

- Provision of games and exercises that can be customised and are appropriate to stroke users. Most games and exercises are often inappropriate for the stroke population (in terms of feedback, length, speed, complexity and range of movement). They have to be adjusted for each patient's specific impairments / limitations [3],[12],[15].

- Patient difficulties in holding the Wii remote due to poor distal limb function [14].

In agreement with these studies, the results from initial stages of the ReWiiRe project found a high level of acceptability for the use of Wii in rehabilitation with $76.4 \%$ of patients $(n=88)$ and $72.1 \%$ of therapists $(n=92)$ agreeing that it was a useful tool in rehabilitation and $75.5 \%$ noting that they would like to use it as part of a HEP using teletherapy. Yet, results also highlighted a number of limitations of use, particularly in the field of neurorehabilitation where the duration, speed and complexity of the exercises, the dexterity required to hold and operate the Wii remote (known colloquially as the Wiimote), the accuracy, sensitivity and ability to pick up small movements (typical of stroke survivors) limited its use clinically.

Several studies have reported customised Wii-based interventions aimed at developing a VRbased system that captures the patient upper limb motion in a more accurate way compared to the conventional Wii remote. These can be categorised into three main groups depending on the technical methodology employed for customising the Wii remote, namely, acceleration data only, Wiimote and light-emitting diodes (LEDs) as an Infra Red (IR) camera and a hybrid of the two. 
The majority of customised Wii remote stroke interventions employ one or two Wiimotes with a reverse engineered application programming interface (API) to capture the patient motion by reading the Wiimote accelerometer data.

A number of algorithms have been suggested and implemented by various research groups to filter these data and map them to motion to drive either a set of exercises or custom-built games, such as [16]-[18]. Since all of these approaches obtain the position of the Wiimote in space using the acceleration data (that is the change in the linear acceleration as the patient moves the Wii remote in space) they suffer from a Degree-of-Freedom (DoF) limitation. More precisely, these solutions offer accuracy only in 2-DoF as the acceleration data can only determine the pitch and roll movement. Such systems are therefore more appropriate for gesture-based interventions rather than one-to-one mapping (kinematic animation) of movement onto the VR environment.

Another common Wiimote customisation involves using a pair of Wiimotes with the LED sensor bar or custom LEDs as an IR camera as a low-cost motion capture system, such as [19]-[21]. Reflective markers or LEDs are usually placed on patient's arm or hand and as the exercises are executed, the range of motion is captured and mapped onto the system display. The limitation in this approach is that each Wiimote can only detect up to four LEDs in space, thus restricting the range of movement and set of exercises that the patient can execute. Also care must be taken to ensure that the angle of the Wiimotes does not cause occlusion of LEDs as the patient moves his/her arm. Moreover, the use of LEDs often requires extra power supply devices to be attached to patients.

Wilson [22] and Martin-Moreno [23] have proposed a hybrid solution that incorporates the two aforementioned approaches in an effort to increase accuracy and the number of DoFs. However, this approach suffers from a limited field of view by employing the Wiimote as an IR camera, making it unsuitable for tracking larger motions that are often required in therapy [18]. Williamson et al [24] have proposed a Wii-based system that merges acceleration and gyroscope data with the Wiimote's IR. This system is aimed at motion recognition for sport-like games (e.g. American football) and not for kinematic analysis and mapping of user motion on a 3D avatar (virtual character).

The data fusion algorithm developed by Williamson et al [24]requires the use of the Wiimote's IR camera to compensate for the gyroscope drift and movement corrections. Results indicate improved motion recognition when compared to acceleration data alone but loss of accuracy when IR is out of sight. 


\section{The ReWiiRe System}

The system described in this paper was, developed within the ReWiiRe project, following an iterative participatory design and development cycle, where results from qualitative research involving patients and therapists were used to ensure the development is fit for purpose.

The system architecture, as depicted in figure 1, consists of two front-end interfaces and a backend database server, namely the patient and the therapist applications and an on-line data-recording platform. It is possible to omit the back-end platform from the infrastructure and maintain a direct connection between the two front-ends, but at the cost of an expandable and reliable system.

Figure 1. ReWiiRe's System Architecture

The software is comprised of three bottom-to-top layers, namely the data access and collection, the data fusion and motion tracking algorithm, and the 3D visualization. The data access operation includes functions that allow the application to acquire sensor data sent from multiple Wiimotes to a personal computer via Bluetooth communication (two Wiimotes are used in our study but more can be added). In the middle layer, the information received from the Wiimote sensors undergoes a process of smoothing and multiplexing using a data fusion algorithm in order to achieve higher accuracy and precision. The end results are mapped into quaternion forms that translate the orientation of a constructed 3D body model and also form the data structure for the top layer. The top layer embraces the dynamic physical simulation of a 3D avatar animation in real-time. For the purpose of the animation, we employ Blender ${ }^{2}$, which is an open source 3D application.

The motion capture information is stored locally and within a predefined time-interval is forwarded to a remote location using secured communication flows (known as network sockets). A database maintains the recorded sessions for each individual patient and is available to the therapist for evaluation and monitoring. It serves as a direct link between the patient and therapist, therefore it can also be used as a communication tool (e.g. the therapist may leave messages for the patient to receive or amend the patient's workout list as (s)he progresses). As the server holds sensitive information, a set of secure protocols is necessary.

\footnotetext{
${ }^{2}$ For Blender's home website, see http://www.blender.org/
} 
The use of network sockets enables interoperability of our data usage in a cross platform environment (bidirectional inter-process communication flow across the host computer and a remote network) and the ability for the therapist to monitor patient progress both off-line (store-and-forward method) but also in real-time (getting a real-time 3D visualisation of the patient's current exercise activities). It should be noted that although the system architecture employs the Nintendo Wii technology for the capture of the motion; it has been designed to be scalable so that other game sensors, such as the Microsoft Kinect, can be employed for the capturing of patient motion.

\section{The Patient Application}

Typically VR environments for upper limb rehabilitation are categorized into game-like or teacheranimation [25]. In the former, the patient accomplishes tasks in the context of a game, whereas in the latter, the patient is directly guided throughout the movement. Our system supports both modes, as from the patient application interface, the user can choose a set of exercises from a predefined exercise library set by the therapist, play customised games or check his / her progress. Furthermore we have designed the intervention as a hands free system (the Wiimotes are strapped on the patient's forearm and upper arm). This is an important aspect of the system, which was developed in response to feedback by stroke therapists in the reWiiRe study, as most stroke survivors cannot grasp and manipulate objects, such as the Wiimote.

To help facilitate the correct execution of the exercises, the design concept of a "Virtual Teacher", a term coined by Holden et al. [26], was applied. see figure 2. By superimposing the teacher's arm movement (therapist in this case), on the patient's arm movement and instructing the patient to follow the teacher's arm trajectory, the patient is more likely to perform the action correctly. The provision of real-time $3 \mathrm{D}$ representation of the arm that is controlled by the patient is a key component of the ReWiiRe system, as it relates feedback to action by directly representing patient's experience in physical reality.

Figure 2. The design of the "Virtual Teacher" concept. The patient needs to follow the red arm to perform the prescribed by the therapist exercise.

Furthermore, the system is designed to be expandable so additional applications and games can be integrated into the exercise library. Figure 3 illustrates the "Hitting the Ball" game, which has been 
designed and piloted within this project. In this game the patient moves his / her arm in order to interact with (e.g. move) the avatar's arm and hit the 3D balls within the VR space for a predefined period of time. Game parameters, such as the time that a new ball randomly appears or how far from the avatar's body it moves, as well as the arm range of motion that the avatar supports are fully adaptable dynamically, in order to cater for each patient's specific needs. The game records the range of movement and score, to provide the therapist with feedback but also reports back to the patient at the end of each session with simple, yet encouraging and positive feedback messages, such as "Well done a new record was made", "Good effort", etc.

Figure 3. The "Ball Hitting" game concept. The patient must hit the balls that appear randomly and within a given time.

\section{The Therapist Application}

The therapist application interface comprises a more multifunction application and has direct access to the database of exercises and games. Initially, the therapist is able to retrieve an individual exercise session and with the aid of the 3D application, (s)he can get a visual presentation of the patient carrying out that specific exercise as if (s)he was present during the execution. Through the application, the therapist is able to prescribe a new set of exercises by either selecting them from an existing library or by creating a new one in a perform-capture-record manner similar to the patient's "Virtual Teacher" interface. An additional function is the ability to review a range of sessions and monitor the progression of the limb's motor function. The results are displayed in units of arc degrees (i.e. elbow extension $=20$ degrees) and percentile improvement from the original assessment (see figure 4). The therapist can also define the range of movement, speed, duration, number of repetitions, scoring and feedback for each set of exercises as well as the prototype games, according to the degree of disability and needs of each stroke patient. Hence the system provides remote progress, assessment and interaction between therapist and patient as well as the ability to customise exercise and game settings to individual patient needs.

Figure 4. The therapist application.

\section{Motion Capture and VR Mapping System}


In electronics, the Wiimote coupled with the Wii Motion Plus (or the recently integrated Wiimote Plus) can be considered as a low-cost, IMU since it features both accelerometer and gyroscope sensor devices. Although, these sensors are highly responsive, like any other Micro-Electro-Mechanical System (MEMS) devices, the readings can be prone to noise and errors, such as bias, drift, angular random walk, etc. A sensible approach to the problem is to process concurrently and conjointly both sensors' measurements through a data fusion algorithm so, raw information captured from one sensor can be used to distinguish true readings of the other, and vice versa. Also, the angular rate measurements captured by the gyroscope sensor can be used to distinguish true linear motion from the accelerometer readings and in combination with a matching human body's kinematic analysis, a highly accurate, well responsive one-to-one representation of the control device in 3D space 6-DoF can be achieved. The goal, with regard to the design of the physical simulation, was to mimic the motions seen in the arm close to real-time correspondence. This would, enable users to feel a connection between their actions and that of the 3D avatar, providing them with instant visual feedback to increase motivation to exercise. The full description and discussion of the Motion Capture (mocap) system is beyond the scope of this paper but more information can be found in [9].

\section{Mocap System Accuracy Validation}

Based on the goal, described above, it is clear that the accuracy of the MoCap system was a major challenge and therefore an experiment was conducted to evaluate this, in which the mocap system was compared with a well-established professional and accurate system, namely the Vicon ${ }^{\mathrm{TM}}$ MoCap Studio $^{3}$. The Vicon ${ }^{\mathrm{TM}}$ MoCap studio comprised of eleven infrared cameras and a MoCap software platform, namely Blade 1.7. The software offers a calibration procedure that matches the camera positioning with the cameras in the 3D environment and reconstructs the actual scene into $3 \mathrm{D}$, hence matching the camera frames with the environment frame. The highly sophisticated MoCap studio is capable of achieving accuracy of sub-milimeters. As the ReWiiRe system is markerless, there was no interference when the two systems worked in parallel. A six-rod structure was built to support the Wiimote and provide reference of position into the Vicon platform. For that reason, one reflective marker was attached at the end of each equidistant rod, and two markers were attached to another rod for reference purposes (see figure 5). The structure was wooden as this material does not have

\footnotetext{
${ }^{3}$ http://www.vicon.com/
} 
any magnetic properties and therefore would not interfere with the measurement units of the Wiimote. The resulting object within the VR environment was an octahedron with the markers acting as vertices. The Wiimote was placed at the centre of the octahedron. As the individual vertices of the octahedron were captured and the motion data was already known, it was possible to calculate the motion data at the centre, which had been designed to be the middle point, as seen on the figures below. The structure was supported by two stands so that it could rotate freely along any axis. All axis were tested by a rotational pattern of $+90,-180,+90$ degrees and included three repetitions each time to compile a dataset that could render the system performance. The recordings of both systems were taken simultaneously for time reference purposes.

Figure 5. The experimental setup of our mocap system.

\section{Mocap Validation Results}

The results presented below are very encouraging as they are very close to the data gathered by the Vicon system, which has an established accuracy level of sub-millimetre.

The movement pattern shows high correlation between the Wiimote and the MoCap systems and quantitative analysis of the Wiimote system data showed a range of accuracy between $5.5 \%-10.78 \%$ residual value $(5.5 \%, 9.39 \%$ and $10.78 \%$ correspondingly for each of the axis depicted in the figures $6,7, \& 8$ below).

Figure 6. Wiimote mocap system accuracy data in comparison to the Vicon platform-X Axis.

Figure 7. Wiimote mocap system accuracy data in comparison to the Vicon platform - Y Axis.

Figure 8. Wiimote mocap system accuracy data in comparison to the Vicon platform - Z Axis.

This value is the Normalised Root-Mean-Square Deviation (NRMSD), which is commonly used as a formula to calculate deviation for such cases as ours where $n=$ number of samples for each recording. 


$$
\begin{aligned}
& \text { NEMSD }=\frac{\text { RMSL }}{x_{\mathrm{m} H x}-x_{m: \Omega}}(1), \\
& \text { where } \mathrm{R} W S D=\sqrt{\frac{\sqrt{\frac{\sum_{L}^{n}-1\left(y_{L}-y_{L}\right)^{2}}{\pi}}}{\pi}}(2) \text {, }
\end{aligned}
$$

The residual values demonstrated that the ReWiiRe system, as depicted in the figure above, has acceptable levels of accuracy when compared to the commercial and expensive MoCap system. This renders it a valid candidate for the purposes of a biofeedback platform. It should be noted, that the measurement error was due to a broad range of reasons; from magnetic fields that affect the performance of the IMUs, to synchronisation errors during the data capture process. The NRMSD values are an indicator that the proposed system will provide results of acceptable accuracy for a home-based and tele-monitoring system. .

\section{Preliminary Evaluation}

This section presents the protocol and results of the preliminary evaluation of the final system and findings from a proof-of-concept case study with a stroke survivor who was 11 years post-insult.

\section{System Evaluation}

\section{Method}

Following ethical approval for the study, three stroke survivors were recruited to the ReWiiRe team and assisted with system evaluation and further development:

- Participant 1 - 33 year old male with right-sided hemiplegia following a stroke 18 months previously. Scored 3 on the modified Rankin Scale (mRS) indicating moderate disability, with moderate gross movement at the shoulder and elbow, flickers at the wrist and hand (no grasp). Had played with the Nintendo Wii prior to his stroke but had not used it since.

- Participant 2 - 52 year old female with right hemiplegia following a stroke 16 months previously. Scored 2 on the mRS indicating slight disability with weak and uncoordinated movement throughout the arm and hand. Had played with the Nintendo Wii prior to her stroke but had not used it since. 
- Participant 3 - 57 year old female with left hemiplegia following a stroke 14 months previously. Scored 4 on the mRS indicating moderately severe disability with minimal movement at the shoulder and elbow and some non-functional movement in the hand. No previous experience with the Nintendo Wii.

Participants took part in three trials using the Wii-based stroke intervention in a university therapy room (see figure 9). All participants were given time to familiarise themselves with the system. The field test, including familiarisation with the protocol, lasted 50 minutes. The trials are summarised below:

1) Teacher animation trial: each participant performed three, pre-recorded upper-limb exercises in which (s)he had to follow a 3D avatar projected on a screen. Three movement patterns, involving the shoulder, elbow and forearm, were selected on the basis of functional utility and subject difficulty in movement performance. The exercises were different for each participant in terms of speed, range of movement and movement performed, dependent on each participant's specific impairments. Each movement pattern was performed by participants for 3 sets of 5 repetitions.

2) Ball hitting game trial: involved the use and display of the 3D avatar on the projector screen where participants tried to "hit" randomly appearing balls, (high, middle, low, central, left or right of the screen avatar). Each ball remained on the screen for 5 seconds, or less if successfully hit. The time taken to hit (or miss) 25 balls was recorded. Two sets of 25 were completed.

3) Air hockey game trial: centred on playing an air hockey game for 5 minutes. This involved moving a "puck" forward, backward and side-to-side through combined movements of the elbow and shoulder to score goals and defend their own "net" against the computer opponent. The range of movement was changed to accommodate the ability of each participant.

All sessions were captured via video and observation notes were made during the trials. After completion of field testing, participants shared their comments, insights and experience about using the customised Wii intervention with the rest of the research team.

Figure 9. Study participant pilot testing the three trials

\section{Results and Discussion}


A number of themes emerged from post intervention debriefs and observation notes, namely: participant connection with the avatar, normal versus mirror view and opinions on the personalisation of the VR environment.

\section{Participant Connection with the Avatar}

Participants reported the importance of being able to see their movement mapped onto a virtual character (avatar), as it helped them in getting a direct visual feedback of the action performed at the time. Participants also reported feeling connected with the avatar:

"I actually like when I am doing it. It (referring to the avatar) feels like that's me" (Participant 1)

This was more evident in the teacher animation and hitting the ball trial. In the former, participants indicated the usefulness in being able to see the physiotherapist's pre-recorded exercise on the screen whilst they were trying to match it. They stressed the importance of feedback and guidance and reported that this would enable the session to take place at home instead of the clinic:

"It [the system] would be like having the physio at home with me, guiding me all the way through what [exercises] I have to do, plus I can see and compare how well I am doing" (Participant 2)

It was observed, particularly in the case of participants 1 and 2, that their ranges of movement improved (i.e. they were able to extend their arms further) when conducting the exercise whilst looking at the avatar compared to exercising without the device. In addition, participants reported that they highly valued the responsiveness of the system, particularly in terms of the visual feedback provided through the depiction of the arm movement on the VR environment:

"It is great! I can see my arm movement appear instantly on screen and can tell how well and far I have gone" (Participant 3) 
Participants were asked to perform the exercises in the teacher animation trial while seated and standing up; the avatar was in both cases in a standing posture. Participant 2 did not mind this but participants 1 and 3 found it less helpful:

"It is easier to do exercises when he (referring to the avatar) is standing, it feels more like me" (Participant 1)

Participant responses indicated a direct link between patient motivation and personal connection with the avatar, especially when combined with instant visual representation and feedback of motion on the screen. This was true with regards to the position of the avatar with participants suggesting that if they are seated, so should the avatar. This was not the case however in terms of the avatar's personal physical characteristics, with all three subjects reporting this as irrelevant.

\section{Default Versus Mirror View}

Another element investigated, in both the teacher animation and hitting the ball game, was the uses of default and mirror views (similar to watching oneself in a mirror). All participants reported preference for the mirror view, as they found it more natural and they were accustomed to using a mirror when conducting exercises either at home or in the gym. It was also observed that participants performed better in terms of response time and scored higher (in the case of the hitting the ball trial) when using the mirror view:

"It feels (referring to the mirror view) a more natural way of seeing things" (Participant 3)

It was not anticipated that viewpoint would have such an impact on participant interaction with the VR environment and performance. It is therefore suggested that any VR stroke rehabilitation environment should support a mirror view, as it is perceived by stroke patients as a more natural way of viewing and understanding their movement. Feedback from this stage resulted in the use of a mirror view being adopted.

\section{VR Environment Personalisation}


According to participants, a key feature of the trialled activities was the ability to personalise various aspects of the VR environment. In particular, exercise duration, range of movement, game-play I activity speed and avatar viewing angle were regarded as the most important personalisation features. Participants commented that when interacting with the teacher animation they found being able to select from a range of prescribed exercises of great benefit. They reported that being able to have control over the range of movement was very important in terms of accessibility.

"I can now play games and do things not possible for me before" (Participant 2)

"I used to play Wii bowling and golf before. This is the first time since then I have played games with the Wii again" (Participant 1)

In addition to this, participants indicated that being able to adjust the speed in the hitting the ball and air hockey games made the tasks more manageable and enjoyable:

"I found it (referring to the hitting ball trial) hard at the beginning, as it was too fast for me. After changing the speed it become easier and I could follow it" (Participant 3)

This was found to be a key factor for the adoption of the trialled activities, as different stroke survivors have very different abilities and response times. For instance participant 3 had the slowest reaction time and was slower in completing the tasks when compared to the others.

\section{Single Case Study}

\section{Method}

A single-case, before and after study was undertaken to field test the system [27]. The participant was a 31 year-old, right-handed, female (BM), who had suffered a right sided stroke 11 years previously, resulting in a left sided hemiplegia (scoring 2 on the mRS). She had finished all formal rehabilitation.

The study took place in a university therapy room, three times weekly over a two-week period with the subject in standing using the same activities and procedure outlined in the previous section. 
Two-minute rest periods were introduced between each exercise and activity. Six sessions of therapy were completed with an average of 18 minutes spent exercising (excluding rest periods).

Outcome measures to assess for any change in motor impairment [Fugl-Meyer Assessment (FMA) upper limb section and Nine Hole Peg Test (NHPT)] [28], spontaneous functional use [Motor Activity Log (MAL) - Amount of Use (AOU) sub-scale] or any increase in muscle tone (spasticity) [Modified Ashworth Scale (MAS)], were used to assess baseline ability and outcome (see table 1). To avoid effects of fatigue, measurements were taken one day prior to the start of the exercise sessions and one day post, by a single assessor. In addition, the subject was asked to subjectively quantify perceived effort and enjoyment of the activities.

\section{Results and Discussion}

Results from the outcome measures are reported in table 1. No adverse effects were reported and positive changes were seen in all outcome measures.

Table 1. Pilot single-case study outcome measures.

A large improvement in score on the NHPT (from 29 to 7.8 seconds per peg) indicated a significant improvement in upper limb impairment. The NHPT requires the patient to pick up and place $9,9 \mathrm{~mm}$ wooden dowels (known as pegs) in a wooden base with $10 \mathrm{~mm}$ holes spaced $15 \mathrm{~mm}$ apart in a $3 \times 3$ arrangement. The time taken to place all pegs is recorded and reported as seconds to place each peg. The participant completed two trials of the NHPT at each measurement session and the results from the two attempts were averaged to provide the final score. The participant dropped two pegs just as she was about to place them in the board on both of the trials before the study (resulting in the assessor retrieving the peg while the participant continued the test with another peg) and this contributed to the poorer score seen before the study. No pegs were dropped in the post study assessment.

A clinically significant increase in score was seen on the FMA (an increase of 8 points from 39-47 on a 66 point scale) suggesting an improvement in impairment post study. Moreover, an improvement in score for functional spontaneous use of the arm (MAL score from 7-10 on a 70 point scale) indicated that the participant was spontaneously employing her affected arm more following the study. 
The MAS is a six-point ordinal scale measuring spasticity or uncontrolled tightening of muscles (where 0 indicates no increase in spasticity and 5 indicates that the limb is held in a rigid posture) and was employed to detect whether use of the technology resulted in an adverse increase in spasticity. The MAS score remained unchanged in the shoulder and elbow and improved slightly (from 3-1) in the finger and wrist flexors indicating a non-significant reduction in spasticity and demonstrating that the use of the system did not result in an adverse increase in spasticity with this subject

The participant described her left upper limb as feeling "warmer", "more muscularly defined", and "lighter". Moreover, she reported that she had greater speed and range of movement and that her "hand wanted to open of its own accord". She also reported better wrist control, greater functional use and noted that she had automatically used her left arm to lift and drain a cooking pan for the first time since her stroke.

While the results from this study are very encouraging, due to the nature of the study, it is not possible to state with certainty that the improvements seen were as a result of the intervention alone, however, at 12 years post stroke, it is unlikely that the participant would have spontaneous recovery which typically occurs within the first three months following stroke.

Figure 10. Stroke survivor pilot testing the "Teacher Animation" prototype.

The activities employed, possessed a high level of user-acceptability which is critical in improving compliance with exercise programmes. The stroke survivor in this case study reported that exercise 1 was only moderately fun but nevertheless enjoyable since it felt like a "good workout" (see figure 10). She expressed great enjoyment of the ball hitting activity as she scored well and hence found this both fun and motivating. However, as she improved, her enjoyment level began to wane, suggesting the need to progress activities to ensure they remain challenging Interestingly, the participant was most interested in trialling the air hockey game as this was something that she had played in reality. However, she initially expressed limited enjoyment due to frustration with her, unexpectedly, low score. With repetition and recalibration of the game speed and range of movement required, her score and motivation improved to the extent where she rated the game as $9 / 10$ for fun; expressed the wish to play for longer periods and described how, in her own words, "the phrase air hockey, caused a Pavlovian response with regard to motivation!" providing further evidence for the need for individual calibration of activities. 


\section{Conclusions}

Over a third of stroke survivors have a long-term disability and many experience reduced quality of life. Compliance with traditional rehabilitation techniques and limited resources to provide therapy particularly on discharge from the hospital, mean that the intensity of practice necessary to drive recovery is often not achieved. This paper has presented an innovative system, developed to offer a customised stroke VR-based rehabilitation intervention by using low-cost off-the-shelf game sensors (the Nintendo Wii remote combined with open source 3D software). The presented system offers full kinematic tracking of the user upper limb movement in real-time, thereby making it possible to have a strong personal connection between a stroke survivor and a virtual character to enhance the clinical utility of this innovative technology. This is the first project to fuse the Nintendo Wii remote acceleration and gyroscope data together to track the controller movements and offer a 6-DoF with the use of the Wii remote alone.

System evaluation with stroke survivors (with varying levels of disability), showed positive results. They reported a strong connection with the system and avatar, mainly due to the real-time visual depiction and feedback of their arm movements on the VR environment. They commented positively on the personalisation features of the system, especially in terms of the exercise range, range of movement and game-play/activity speed. These features enabled them to engage in activities and games otherwise unsuitable for them. For some participants, it was the first time they had interacted with VR gaming environment since their stroke. It was also found that use of a mirror view when performing exercises increased stroke patient connection with the VR environment, as well as response times and performance.

Our preliminary clinical trial results also indicated positive changes in the four outcome measures employed in our single-case study. Despite the short study duration, significant improvements were demonstrated, particularly in the NHPT and MAL where significant improvements were observed and greater functional use of the affected arm was reported. It was also noted that activities employed through the customised Wii system were fun and motivating and that customisation of the activities, such as the speed and range of movement required, increased the stroke survivor's score and motivation. It is interesting to note that the case study subject demonstrated improvement in hand 
function despite the device not actually targeting the hand. This may be due to muscles in the forearm also being involved in hand movement.

Encouraged by the preliminary results, the team plan to extend the study in terms of duration and participant numbers. In addition, exploration of the feasibility and acceptability of the system with community dwelling stroke survivors and studies exploring the use of the system in other neurological conditions are planned.

\section{Acknowledgements}

The authors would like to acknowledge the National Health Service (NHS) London Regional Innovation Fund, which supported the ReWiiRe project, all the stroke survivors who took place in the system evaluation and development and our NHS project partners.

\section{Declaration of Interest}

The authors report no declarations of interest.

\section{References}

[1]Morse A, General A. Department of Health: Progress in improving stroke care. London; 2010 p. 39. Available from: http://www.publications.parliament.uk/pa/cm200910/cmselect/cmpubacc/405/405.pdf

[2]Hendricks $\mathrm{H}$. Systematic review for the early prediction of motor and functional outcome after stroke by using motor-evoked potentials. Archives of Physical Medicine and Rehabilitation. 2002;83(9):1303-1308.

[3]Lewis GN, Woods C, Rosie $\mathrm{J}$ a, McPherson KM. Virtual reality games for rehabilitation of people with stroke: perspectives from the users. Disability and rehabilitation. Assistive technology [Internet]. 2011 January [cited 2013 June 3];6(5):453-63. Available from: http://www.ncbi.nlm.nih.gov/pubmed/21495917

[4]Lu EC, Wang RH, Hebert D, Boger J, Galea MP, Mihailidis A. The development of an upper limb stroke rehabilitation robot: identification of clinical practices and design requirements through a survey of therapists. Disability and rehabilitation. Assistive technology [Internet]. 2011 January [cited 2014 June 11];6(5):420-31. Available from: http://www.ncbi.nlm.nih.gov/pubmed/21184626

[5]Miyoshi T, Takahashi Y, Lee H, Suzuki T, Komeda T. Upper limb neurorehabilitation in patients with stroke using haptic device system: reciprocal bi-articular muscle activities reflect as a result of improved circle-drawing smoothness. Disability and rehabilitation. Assistive technology [Internet]. 2010 January [cited 2014 June 11];5(5):370-5. Available from:

http://www.ncbi.nlm.nih.gov/pubmed/20131971

[6]King M, Hale L, Pekkari A, Persson M, Gregorsson M, Nilsson M. An affordable, computerised, table-based exercise system for stroke survivors. Disability and Rehabilitation: Assistive Technology 
[Internet]. 2010 July [cited 2014 June 11];5(4):288-93. Available from:

http://www.ncbi.nlm.nih.gov/pubmed/20302419

[7]Glegg SMN, Tatla SK, Holsti L. The GestureTek virtual reality system in rehabilitation: a scoping review. Disability and rehabilitation. Assistive technology [Internet]. 2014 March [cited 2014 June 11];9(2):89-111. Available from: http://www.ncbi.nlm.nih.gov/pubmed/23713408

[8]Mawson S, Nasr N, Parker J, Zheng H, Davies R, Mountain G. Developing a personalised selfmanagement system for post stroke rehabilitation; utilising a user-centred design methodology. Disability and rehabilitation. Assistive technology [Internet]. 2013 October 16 [cited 2014 June 11]. Available from: http://www.ncbi.nlm.nih.gov/pubmed/24131371

[9]Tsekleves E, Skordoulis D, Paraskevopoulos I, Kilbride C. Wii Your Health: A Low-Cost Wireless System for Home Rehabilitation after Stroke using Wii Remotes with its Expansions and Blender. In: Proceedings of Biomedical Engineering. ; 2011. Available from: http://www.actapress.com/PDFViewer.aspx?paperld=451737

[10]Saposnik G, Teasell R, Mamdani M, Hall J, Mcllroy W, Cheung D, Thorpe KE, Cohen LG, Bayley $M$. Effectiveness of virtual reality using Wii gaming technology in stroke rehabilitation: a pilot randomized clinical trial and proof of principle. Stroke; a journal of cerebral circulation [Internet]. 2010 July [cited 2013 May 21];41(7):1477-84. Available from:

http://www.ncbi.nlm.nih.gov/pubmed/20508185

[11]Reinkensmeyer DJ, Boninger ML. Technologies and combination therapies for enhancing movement training for people with a disability. Journal of neuroengineering and rehabilitation [Internet]. 2012 January [cited 2014 April 30];9(1):17. Available from:

http://www.pubmedcentral.nih.gov/articlerender.fcgi?artid=3349545\&tool=pmcentrez\&rendertype=abs tract

[12]Levac D, Miller P, Missiuna C. Usual and Virtual Reality Video Game-based Physiotherapy for Children and Youth with Acquired Brain Injuries. Physical \&amp; Occupational Therapy in Pediatrics [Internet]. 2012;32(2):180-195. Available from: http://dx.doi.org/10.3109/01942638.2011.616266

[13]Faria C, Silva J, Campilho A. Rehab@home: a tool for home-based motor function rehabilitation. Disability and rehabilitation. Assistive technology [Internet]. 2013 September 26 [cited 2014 June 11]. Available from: http://www.ncbi.nlm.nih.gov/pubmed/24070452

[14]Lange B, Koenig C, Chang C, McConnell E, Suma M, Bolas M, Rizzo A. Designing informed game-based rehabilitation tasks leveraging advances in virtual reality. Disability and rehabilitation. 2012;34(22):1863-1870.

[15]Parker J, Mountain G, Hammerton J. A review of the evidence underpinning the use of visual and auditory feedback for computer technology in post-stroke upper-limb rehabilitation. Disability and rehabilitation. Assistive technology [Internet]. 2011 January [cited 2014 May 29];6(6):465-72. Available from: http://www.ncbi.nlm.nih.gov/pubmed/21314295

[16]Leder R, Azcarate G. Nintendo Wii remote for computer simulated arm and wrist therapy in stroke survivors with upper extremity hemipariesis. In: Virtual Rehabilitation. Vol. 10. Vancouver, Canada; 2008. p. 47. Available from: http://ieeexplore.ieee.org/xpls/abs_all.jsp?arnumber=4625137

[17]Palmke M, von Piekartz H, Zalpour C, Schuler T, Morisse K. A new perspective for Virtual Mirror Therapy. In: Virtual Rehabilitation International Conference. Vol. 88. ; 2009. p. 4244.

[18]Alankus G, Proffitt R. Stroke therapy through motion-based games: a case study. In: 12th international ACM SIGACCESS conference on Computers and accessibility (ASSETS '10). ACM; 2010. pp. 219-226. Available from: http://dl.acm.org/citation.cfm?id=2039342 
[19]Jovanov E, Hanish N, Courson V, Stidham J, Stinson H, Webb C, Denny K. Avatar - a multisensory system for real time body position monitoring. Conference proceedings : ... Annual International Conference of the IEEE Engineering in Medicine and Biology Society. IEEE Engineering in Medicine and Biology Society. Conference [Internet]. 2009 January;2009:2462-5. Available from: http://www.ncbi.nlm.nih.gov/pubmed/19964961

[20]Milosevic M, Jovanov E. A real-time control of multiple Avatars using Wii remotes and Avatar system. In: 2011 IEEE 43rd Southeastern Symposium on System Theory. leee; 2011. pp. 139-142. Available from: http://ieeexplore.ieee.org/lpdocs/epic03/wrapper.htm?arnumber=5753793

[21]Standen PJ, Brown DJ, Battersby S, Walker M, Connell L, Richardson A, Platts F, Threapleton K, Burton A. A study to evaluate a low cost virtual reality system for home based rehabilitation of the upper limb following stroke. International Journal on Disability and Human Development [Internet]. 2011 January 1 [cited 2014 May 21];10(4):337-341. Available from:

http://www.degruyter.com/view/j/ijdhd.2011.10.issue-4/ijdhd.2011.063/ijdhd.2011.063.xml

[22]Wilson PH, Duckworth J, Mumford N, Eldridge R, Guglielmetti M, Thomas P, Shum D, Rudolph H. A virtual tabletop workspace for the assessment of upper limb function in Traumatic Brain Injury (TBI). 2007 Virtual Rehabilitation [Internet]. 2007 September:14-19. Available from:

http://ieeexplore.ieee.org/lpdocs/epic03/wrapper.htm?arnumber=4362122

[23]Martin-Moreno J, Ruiz-Fernandez D, Soriano-Paya A, Jesus Berenguer-Miralles V. Monitoring 3D movements for the rehabilitation of joints in physiotherapy. In: Conference proceedings : ... Annual International Conference of the IEEE Engineering in Medicine and Biology Society. IEEE Engineering in Medicine and Biology Society. Conference. Vol. 2008. ; 2008. pp. 4836-9. Available from: http://www.ncbi.nlm.nih.gov/pubmed/19163799

[24]Williamson B, Wingrave C, LaViola J. Realnav: Exploring natural user interfaces for locomotion in video games. In: IEEE Symposium in 3D User Interfaces (3DUI). IEEE; 2010. pp. 3-10.

[25]Lehrer N, Chen Y, Duff M, Wolf S, Rikakis T. Exploring the bases for a mixed reality stroke rehabilitation system, part ii: Design of interactive feedback for upper limb rehabilitation. Journal of neuroengineering and rehabilitation. 2011;8(1):54.

[26]Holden M, Dyar T. Virtual Environment Training: A New Tool for Neurorehabilitation. JournalofNeurologicPhysicalTherapy. 2002;26(2):62-71.

[27]Betker AL, Szturm T, Moussavi ZK, Nett C. Video game-based exercises for balance rehabilitation: a single-subject design. Archives of physical medicine and rehabilitation [Internet]. 2006 August [cited 2014 May 6];87(8):1141-9. Available from:

http://www.ncbi.nlm.nih.gov/pubmed/16876562

[28]Duncan PW, Jorgensen HS, Wade DT. Outcome Measures in Acute Stroke Trials : A Systematic Review and Some Recommendations to Improve Practice. Stroke [Internet]. 2000 June 1 [cited 2014 May 21];31(6):1429-1438. Available from:

http://stroke.ahajournals.org/cgi/doi/10.1161/01.STR.31.6.1429 